\title{
Electronic Structure of AIP Under Pressure Using Semiempirical Method
}

\author{
Hamad R. Jappor ${ }^{1}$, Mudar A. Abdulsattar ${ }^{2}$ and Ahmed M. Abdul-lettif ${ }^{3, *}$ \\ ${ }^{I}$ College of Education, University of Babylon, Hilla, Iraq \\ ${ }^{2}$ Ministry of Science and Technology, Baghdad, Iraq \\ ${ }^{3}$ College of Science, University of Babylon, Hilla, Iraq
}

\begin{abstract}
The effect of pressure on the structural and electronic parameters of zinc-blende aluminum phosphide crystal has been investigated using the large unit cell within the framework of complete neglect of differential overlap and the linear combination of atomic orbital approximation. Cohesive energy, indirect band gap, valence bandwidth, conduction bandwidth, bulk modulus, and valence charge distribution are all obtained. The calculations show a good agreement of lattice constant, cohesive energy, valence bandwidth, and bulk modulus with the experimental data. The calculated band gap is twice the experimental value. This high value of the band gap is expected in Hartree-Fock method. The effect of pressure on the aforementioned properties is investigated. It is found that the indirect band gap, valence bandwidth, bulk modulus and cohesive energy increase with increasing pressure, while the conduction bandwidth decreases. The maximum value of pressure is taken to be $9 \mathrm{GPa}$, because beyond this value, the phase of AlP transforms from zinc blende phase to nickel arsenic phase.
\end{abstract}

PACS: 71.10-w, 71.15-m, 71.15. Ap, 71.15. Nc, 64.10.+h.

Keywords: Pressure, band structure, AlP, semiempirical, LUC, CNDO.

\section{INTRODUCTION}

Aluminum phosphide (AlP) is a wide-indirect band gap semiconductor. At normal conditions, AlP crystallizes in the zinc-blende (zb) structure [1]. High-pressure experiments on this compound are difficult because of sample handling problems; AlP is unstable in air [2]. The $\mathrm{zb}$ form has been reported theoretically to be metastable. The zinc- blende phase is known to transform to the nickel arsenic (NiAs) phase at about ((17 - 9.5 GPa [3]. Although other studies have placed this transformation at a somewhat smaller pressure (7 - 9.3) GPa [4]. At a pressure of about $36 \mathrm{GPa}$ the NiAs phase has been reported to undergo a $\mathrm{Cmcm}-$ like distortion with no significant change in volume. The $\mathrm{CsCl}$ phase is a possible candidate for AlP at very high pressures [2]. AlP is a subject of extensive theoretical studies ranging from the semiempirical to the first principles methods [5] within the density functional theory (DFT) framework using both pseudopotential [2], and all-electron approaches. For the bulk phase of AlP, theoretical calculations based on the Hartree-Fock [6], and potential model [7] have obtained a very good description of its structural and electronic properties.

Over the last few years, the study of materials under high pressure has become an extremely important subject. This is primarily due to both theoretical and experimental developments, which have facilitated such work [8].

*Address correspondence to this author at the College of Science, University of Babylon, Hilla, Iraq; Tel: 009647801106357 ;

E-mail: abdullettif@yahoo.com
The pressure is a continuously varying parameter that can be used in systematic studies of the properties of solids as a function of interatomic distances. An interesting phenomenon that may occur at the applied pressure is a sudden change in the arrangement of the atoms, i.e., a structural phase transition of atomic arrangement. The ultimate pressures in the experiment can lead to a reduction in the volume by a factor of two causing enormous changes in the interatomic bonding [9].

In the present work we study the band structure and some physical properties of cubic AlP under pressure using large unit cell method within complete neglect of differential overlap (LUC-CNDO) method $[10,11]$. This method has been chosen in the present work rather than other methods because this can be used to give reliable and precise results with relatively short time.

\section{CALCULATIONS}

We have used the large unit cell within complete neglect of differential overlap (LUC-CNDO) method in the linear combination of atomic orbital (LCAO) approximation [10] to obtain a self-consistent solution for the valence electron energy spectrum. The iteration process was repeated until the calculated total energy of crystal converged to less than $1 \mathrm{meV}$. The calculations are carried out, on the 8-atom LUC. The positions of atoms that constitute this LUC are calculated in the program according to the zinc-blende structure for a given lattice constant. There are four electrons in average per each atom. Hence we have (32) eigenstates, two 
electrons per state, half are filled (valence band) leaving the other half empty (conduction band) in the ground state. We obtained the energy minima against lattice constant variation.

The basic idea of the large unit cell is in computing the electronic structure of the unit cell extended in a special manner at $\mathbf{k}=0$ in the reduced Brillouin zone ( $\mathbf{k}$ is the lattice wave vector). Using the linear combination of atomic orbitals LCAO, the crystal wavefunction in the LUC-CNDO formalism is written in the following form [12]:

$\psi_{a}(\mathbf{k}, r)=\sum_{u}^{\text {cells basis }} \sum_{p} \exp \left(i \mathbf{k} R_{u}\right) C_{p a}(\mathbf{k}) \varphi_{p}\left(r-R_{u}\right)$

where $C_{p a}$ are the orbital expansion coefficients, the $\mathrm{R}_{\mathrm{u}}$ is the lattice translation vector, and $r$ is a position vector. The atomic orbitals used for the LCAO procedure form the basis set of the calculation. We expand the wave function in a set of Slater-type orbitals (STO), that have the radial form [13]:

$R_{n l}(r)=\frac{(2 \zeta)^{n+1 / 2}}{(2 n !)^{1 / 2}} r^{n-1} \exp (-\zeta r)$

where $\zeta$ the orbital exponent. The expectation value of the electronic energy is:

$\varepsilon=\langle\Psi|H| \Psi\rangle /\langle\Psi \mid \Psi\rangle$

The Hamiltonian for a microcrystal consisting of $\mathrm{N}$ electrons may be written as:

$$
\begin{aligned}
& H=\sum_{a}^{N}\left(-\frac{1}{2} \nabla_{a}^{2}-\sum_{A}^{n_{A}} Z_{A} r_{A A}^{-1}\right) \\
& -\frac{1}{2} \sum_{a}^{N} \sum_{p}^{N} r_{a p}^{-1}+\sum_{A}^{n_{A}} \sum_{B}^{n_{B}} Z_{A} Z_{B} R_{A B}^{-1}
\end{aligned}
$$

where $Z_{A}$ is the core charge, $R_{A B}$ is the distance between the atoms $\mathrm{A}$ and $\mathrm{B}$, and the summation is over all nuclei. The Roothaan-Hall equations can be obtained [14]:

$$
\sum_{p}\left(F_{p q \mathbf{k}}-\varepsilon_{a} S_{p q \mathbf{k}}\right) C_{p q \mathbf{k}}=0
$$

$\mathrm{F}_{\mathrm{pqk}}$ represents the Fock matrix elements. $\mathrm{S}_{\mathrm{pq}}$ is the overlap integral for atomic function $\Phi_{q}$ and $\Phi_{P}$, and can be written as [12]:

$S_{p q \mathbf{k}}=\sum_{u}\left\langle\varphi_{p}\left(r-R_{0}\right) \mid \varphi_{q}\left(r-R_{u}\right)\right\rangle \exp \left(i \mathbf{k} R_{u}\right)$

The Fock matrix elements may represent the sum of the one- and two- electron components:

$$
\begin{aligned}
& F_{o p, u q}=\left\langle\varphi_{p}^{1}(1)\left|-\frac{1}{2} \nabla_{1}^{2}-\sum_{a} Z_{a} r_{1 a}^{-1}\right| \varphi_{q}^{u}(1)\right\rangle \\
& +\sum_{v, \lambda}^{\text {cell basis }} \sum_{r s}^{v \lambda} P_{r s}^{v \lambda}\left(\left(\varphi_{p}^{o} \varphi_{q}^{u} \mid \varphi_{r}^{v} \varphi_{s}^{\lambda}\right)-\frac{1}{2}\left(\varphi_{p}^{o} \varphi_{r}^{v} \mid \varphi_{s}^{\lambda} \varphi_{q}^{u}\right)\right)
\end{aligned}
$$

$P_{r s}^{v \lambda}$ is the density element with the form:

$$
P_{r s}^{v \lambda}=2 \sum_{k^{\prime}} \sum_{a}^{o c c} C_{r a \mathbf{k}^{\prime}}^{*} C_{s a \mathbf{k}^{\prime}} \exp i \mathbf{k}^{\prime} .\left(R_{\lambda}-R_{v}\right)
$$

In equation (5) if $\underline{\mathbf{k}}=0$ then

$\sum_{p}\left(F_{p q 0}-\varepsilon_{a} S_{p q 0}\right) C_{p q 0}=0$

The Fock matrix elements in their final forms in the LUC-CNDO formalism are used in this work to be [11]:

$$
\begin{aligned}
F_{p p}(0)= & -\frac{1}{2}\left(I_{p}+A_{p}\right)+\frac{1}{2} \gamma_{A A}^{00} \\
& +\sum_{B}\left(\mathrm{P}_{B}(0)-Z_{B}\right) \sum_{v} \gamma_{A B}^{0 v}+\sum_{v \neq 0} \beta_{A B}^{0 v} S_{0, v p} \\
& -\frac{1}{2} \sum_{v \neq 0} P_{p p}(0) f(x) \gamma_{A A}^{o v} \\
F_{p q(0)}= & \sum_{v} \beta_{A B}^{0} S_{o p, v p}-\frac{1}{2} P_{p q}(0) \sum_{v} f(x) \gamma_{A B}^{o v}
\end{aligned}
$$

For $\mathrm{p}$ and $\mathrm{q}$ on the same atomic center, $\beta_{A B}$ is the bonding parameter and $\gamma_{A B}$ is the average electrostatic repulsion between any electron on atom A and any electron on atom B, and can be written as

$$
\gamma_{A B}=\iint \varphi_{p}(1) \varphi_{q}(1) \frac{1}{r_{12}} \varphi_{p}(2) \varphi_{q}(2) d \tau_{1} d \tau_{2}
$$

$I_{p}$ and $A_{P}$ are the ionization potential and electron affinity respectively, and $f(x)$ is the modulating function that is given by [15]

$f(x)=\left(\frac{\sin (x)}{x}\right)^{2}$

For the eight atoms LUC $\mathrm{x}$ is given by

$$
x=\frac{\pi R_{A B}}{a}
$$

$\mathrm{R}_{\mathrm{AB}}$ is the distance between the atom $\mathrm{A}$ at the central lattice $\mathrm{o}$ and the atom $\mathrm{B}$ at the $\mathrm{v}$ lattice.

In our calculations, we have treated only valence orbitals of $\mathrm{Al}\left(3 \mathrm{~s}^{2} 3 \mathrm{p}^{1}\right)$ and $\mathrm{P}\left(3 \mathrm{~s}^{2} 3 \mathrm{p}^{3}\right)$. The coordinates of the $\mathrm{P}$ atoms are chosen to be $(0,0,0) ;(0,1 / 2,1 / 2) ;(1 / 2,0,1 / 2) ;(1 / 2,1 / 2$, 0 ) whereas the coordinates of the $\mathrm{Al}$ atoms are chosen to be $(1 / 4,1 / 4,1 / 4) ;(1 / 4,3 / 4,3 / 4) ;(3 / 4,1 / 4,3 / 4) ;(3 / 4,3 / 4,1 / 4)$.

\section{RESULTS AND DISCUSSION}

\subsection{Choice of Parameters}

The number of parameters in the LUC-CNDO method is four. These are the orbital exponent $(\zeta)$, the bonding parameter $(\beta)$, the electronegativity of s-orbital $\left(\mathrm{E}_{\mathrm{s}}\right)$, and the electronegativity of p-orbital $\left(E_{p}\right)$. The value of the orbital exponent determines the charge distribution of electrons around the nucleus in the solid

These parameters are varied firstly to give nearly an exact value of the equilibrium lattice constant, cohesive energy, 
indirect bandgap and valence bandwidth. The remaining of the output data of the programs is a result of the theory that is used in the present work. We found that the investigated properties are sensitive to the aforementioned parameters. The parameters used for AlP in the present work are summarized in Table $\mathbf{1}$.

Table 1. The Adjusted Parameters for AIP in the Zinc-Blende Structure

\begin{tabular}{|c|c|c|}
\hline Parameter & Al & P \\
\hline \hline$\zeta_{3 \mathrm{~s}, 3 \mathrm{p}}(\mathrm{a} . \mathrm{u})^{-1}$ & 1.4 & 2.0 \\
\hline$\beta(\mathrm{eV})$ & -6.0 & -4.82 \\
\hline $\mathrm{E}_{3 \mathrm{~s}}(\mathrm{eV})$ & 9.75 & 24.58 \\
\hline $\mathrm{E}_{3 \mathrm{p}}(\mathrm{eV})$ & 8.68 & 12.086 \\
\hline
\end{tabular}

\subsection{The Electronic and Structural Properties}

The second step after the choice of parameters is to examine the structural properties of AlP at the equilibrium lattice constant in order to test the accuracy of the cohesive energy, indirect bandgap, and valence bandwidth.

Based on the total energy result, we obtained the cohesive energy $\left(\mathrm{E}_{\mathrm{coh}}\right)$ as follows:

$\mathrm{E}_{\mathrm{coh}}=\mathrm{E}_{\text {tot }} / 8-\mathrm{E}_{\text {free }}-\mathrm{E}_{0}$

where $E_{\text {tot }}$ is the total energy, $E_{\text {free }}$ is the free atom sp shell energy, and $\mathrm{E}_{0}$ is the zero-point vibration energy. In the present work $E_{\text {free }}=115.045 \mathrm{eV}$, and this value is taken from the ionization potential of AlP, $\mathrm{E}_{0}=0.057 \mathrm{eV}$, is calculated by the formula $\mathrm{E}_{0}=(9 / 8) \mathrm{k}_{\mathrm{B}} \Theta_{\mathrm{D}}$ (per atom) with $\Theta_{\mathrm{D}}$ is the Debye temperature [16], which is equal to $588 \mathrm{~K}$ [17].

The present value of the cohesive energy is in good agreement with the experimental and other calculations $[16,18,19]$ as shown in Table 2. Fig. (1) displays the total energy versus the lattice constant for AlP. The curve is fitted to the equation of state of Murnaghan [20] from which we obtained the equilibrium lattice parameter $\left(a_{0}\right)$, the bulk modulus $\mathrm{B}$ and its derivative $\mathrm{B}_{\mathrm{O}}{ }_{\mathrm{O}}$, and the cohesive energy as listed in Table 2.

The calculated structural properties of AlP, in comparison with experimental results and other computational results, are shown in Table 2 . We notice that the lattice parameter for the $\mathrm{zb}$ of AlP of $5.453 \AA$ is in good agreement with the experimental value of $5.451 \AA[6]$ with an accuracy of $0.04 \%$. For the bulk modulus, the accuracy is about $0.2 \%$. Table 2 shows that the calculated value of $B_{0}$ is in fair agreement with the previous computational $[4,19]$ and experimental [18] results.

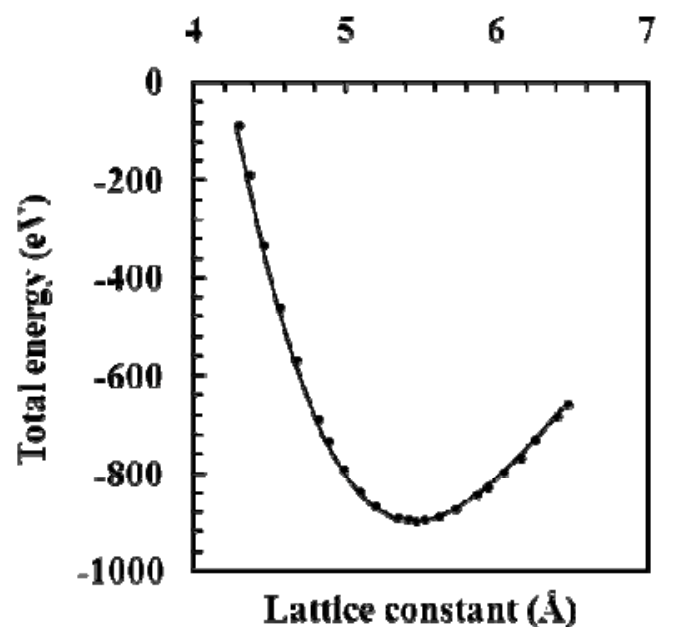

Fig. (1). The total energy as a function of lattice constant for AlP.

To visualize the nature of the bond character and to explain the charge transfer and the bonding properties of zb-AlP, we calculate the total charge density. The total valence charge densities for AlP are displayed along the Al-P bonds in the (100), (110), (200), and (400) planes in Fig. (2). From this figure, it is apparent that the phosphorous ions are larger than the aluminum ions. This figure also shows the charge density accumulated at the P site. Fig. (2a) shows the charge density of the (100) plane, where a buildup of charge density along the AlP bond on the plane is clearly visible. This figure reveals that LUC-CNDO calculations give a reasonable description of the partially ionic bonding character in regions between atoms of different affinities.

\subsection{The Band Structure and Energy Eigenvalues}

The electronic band structure of a solid shows the eigenvalues associated with the valence and conduction bands along specific directions in the Brillouin zone. We will start our discussion of calculations on the band structure by the energy eigenvalues for AlP crystal at various high symmetry points of the Brillouin zone. The results are listed in Table 3. Eight atom LUC results $\Gamma$ and X points of the FCC Brillouin

Table 2. Structural Properties of zb-AIP at Zero Pressure Determined by LUC-CNDO Compared to other Theoretical Calculations and Experimental Data

\begin{tabular}{|c|c|c|c|}
\hline $\mathrm{A}_{0}(\AA)$ & 5.453 & $5.45[2], 5.436[19]$ & $5.451[6]$ \\
\hline $\mathrm{B}(\mathrm{GPa})$ & 87.8 & $86.5[4], 89[19]$ & $90[2], 86[6]$ \\
\hline
\end{tabular}



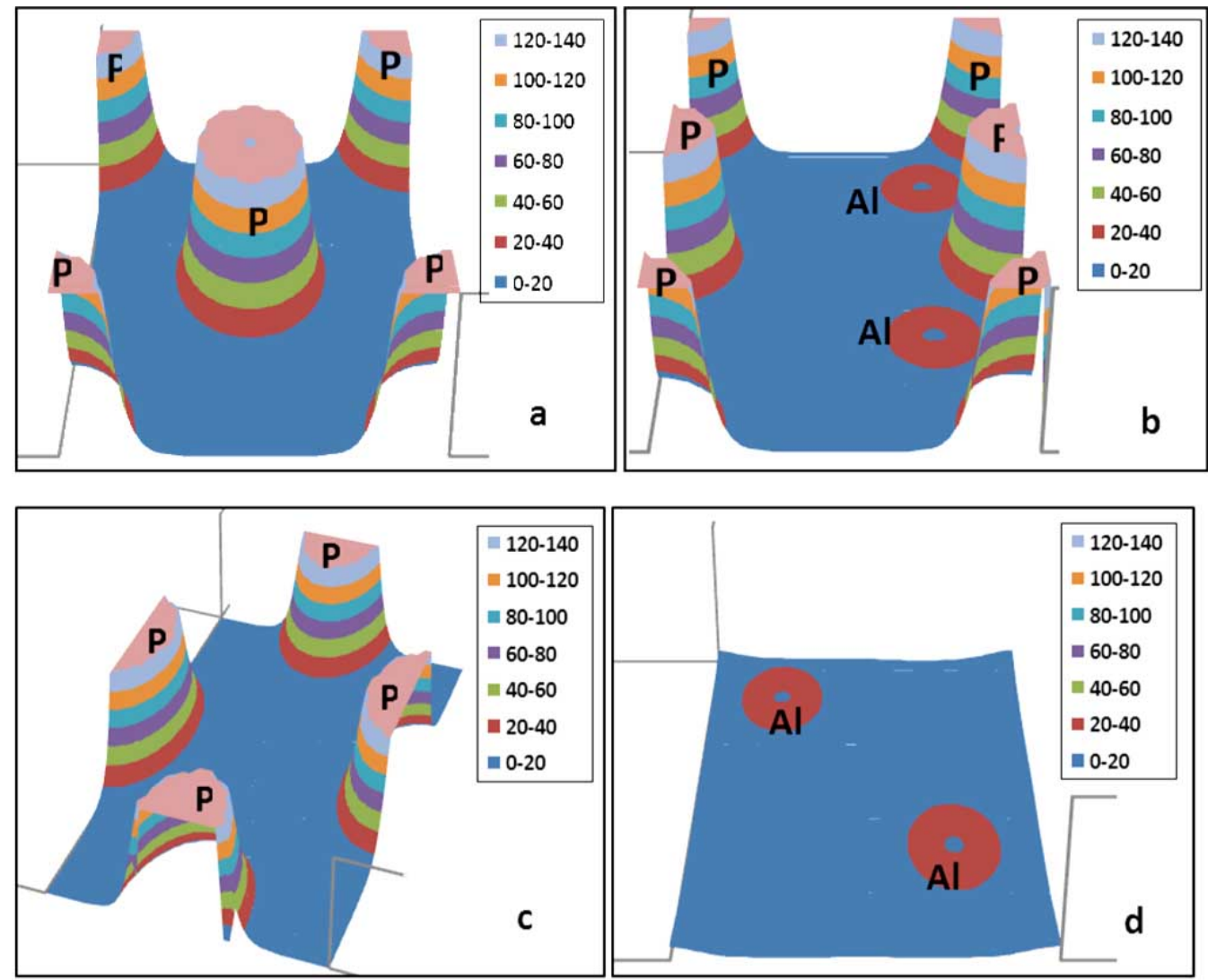

Fig. (2). The valence charge density (in electron $\left./ \AA^{3}\right)$ of zb-AlP at zero pressure in (a) (100) plane, (b) (110) plane, (c) (200) plane, and (d) (400) plane.

zone. They are the valence band states $\Gamma_{1 \mathrm{v}}, \Gamma_{15 \mathrm{v}}, \mathrm{X}_{1 \mathrm{v}}$, and $\mathrm{X}_{5 \mathrm{v}}$, and the conduction band states $\mathrm{X}_{1 \mathrm{c}}, \mathrm{X}_{5 \mathrm{c}}, \Gamma_{15 \mathrm{c}}$, and $\Gamma_{1 \mathrm{c}}$.

The direct bandgap in the zb structures of AlP is due to the $\mathrm{Al} 3 \mathrm{~s}$ and $\mathrm{P} 3 \mathrm{~s}$ orbital interaction, which forms the lowerenergy bonding state $\left(\Gamma_{15 \mathrm{v}}\right)$ and the antibonding state $\left(\Gamma_{1 \mathrm{c}}\right)$. The bonding state is lowered and antibonding states is risen, relative to the $\mathrm{P} 3 \mathrm{~s}$ and $\mathrm{Al} 3 \mathrm{~s}$ orbital energies, by the same amount of s-s interaction energy in AlP. In zb-AlP the conduction-band minimum (CBM) is located away from the $\Gamma$ point, at the $X$ point. AlP has an indirect minimum gap with the CBM at $X$. Thus, we obtain an indirect bandgap ( $\mathrm{E}_{\mathrm{g}}$ ind $)$ of $6.6075 \mathrm{eV}$ which is larger than the results of others [21, 22] (see Table 4 for comparison), this is, mainly, a consequence of two approximations made in the present calculations. First, the core structure was ignored, through some compensation results from using semiempirical parameters. Second, using minimal basis set atomic orbitals (without considering any excited levels). However, the CNDO method predicts a one-electron eigenvalue band gap that is too large and conduction band that is much narrower than the band model values.

The total valence bandwidth (VBW) or the difference between the top of the valence band $\left(\Gamma_{15 \mathrm{v}}\right)$ and the lowest energy of valence band $\left(\Gamma_{1 \mathrm{v}}\right)$ is $13.492 \mathrm{eV}$ for $\mathrm{zb}-\mathrm{AlP}$. The obtained total valence bandwidth is in fair agreement with previous results as shown in Table 4. The maximum level of the valence band is splitted only by spin-orbit interaction $\Delta_{\mathrm{so}}$, giving rise to two states at the Brillouin zone centre: $\Gamma_{8 \mathrm{v}}$ and
$\Gamma_{7 \mathrm{v}}$. In the absence of spin orbit splitting, these levels become a triply degenerated $\Gamma_{15 \mathrm{v}}$. The spin-orbit interaction $\Delta_{\text {so }}$ is taken into account in this paper, averaging the theoretical results from different Refs. to be $0.06 \mathrm{eV}$ [21, 23, 24]. Therefore, we added the relativistic correction to the band gap, which is equal to 0.019 [23]. Our work gives a value of the conduction bandwidth (CBW) to be $14.728 \mathrm{eV}$ (Table 4), but no experimental results are found to the $\mathrm{CBW}$ of the $\mathrm{zb}$ of AlP.

\section{THE EFFECT OF PRESSURE ON THE PHYSICAL PROPERTIES}

The effect of pressure on the electronic structure and other properties can be calculated from the present computational procedure. By the use of our calculated values of the bulk modulus $\mathrm{B}$ and its derivative $\mathrm{B}^{\prime}{ }_{\mathrm{O}}$, the volume change (V) with applied pressure was calculated using the following equation [26]:

$\mathrm{P}=\frac{\mathrm{B}_{0}}{\mathrm{~B}_{0}^{\prime}}\left[\left(\frac{V_{0}}{V}\right)^{\mathrm{B}_{0}^{\prime}}-1\right]$

$\mathrm{P}$ is the pressure and $\mathrm{V}_{0}$ is the equilibrium volume at zero pressure. We use pressures up to $9 \mathrm{GPa}$, because this structure transforms to another phase, the nickel arsenic phase (NiAs), when pressure exceeds nearly $9 \mathrm{GPa}$ [27]. The calculated lattice constant as a function of pressure is shown in Fig. (3). 
Table 3. Eigenvalues (in Electron Volts) at $\Gamma$ and $X$ High-Symmetry Points of Brillouin Zone

\begin{tabular}{|l|l|l|l|}
\hline & Present Work & Computational & Experimental \\
\hline \hline$\Gamma_{1 \mathrm{v}}$ & -13.492 & $-11.46[6]$ & -- \\
\hline$\Gamma_{15 \mathrm{v}}$ & 0.0 & 0.0 & 0.0 \\
\hline$\Gamma_{1 \mathrm{c}}$ & 7.206 & $3.25[6]$ & -- \\
\hline$\Gamma_{15 \mathrm{c}}$ & 8.187 & -- & -- \\
\hline $\mathrm{X}_{\mathrm{lv}}$ & -12.925 & $-9.73[6]$ & --- \\
\hline $\mathrm{X}_{5 \mathrm{v}}$ & -12.921 & -- & -- \\
\hline $\mathrm{X}_{\mathrm{lc}}$ & 6.662 & $1.51[6]$ & -- \\
\hline $\mathrm{X}_{5 \mathrm{c}}$ & 21.39 & - & 3.63 \\
\hline
\end{tabular}

Table 4. Calculated LUC-CNDO Indirect Band Gap, Valence Bandwidth, and Conduction Bandwidth of AIP Compared to other Theoretical Calculations and Experiments. All Energies are in $\mathrm{eV}$

\begin{tabular}{|l|l|l|c|}
\hline & Present & Computational & Experimental \\
\hline \hline $\mathrm{E}_{\mathrm{g}}$ ind & 6.6075 & $3.73[7], 2.77,2.86[25]$ & $3.63[21], 2.45[22]$ \\
\hline $\mathrm{VBW}$ & 13.492 & $11.46[6]$ & --- \\
\hline $\mathrm{CBW}$ & 14.728 & -- & -- \\
\hline
\end{tabular}

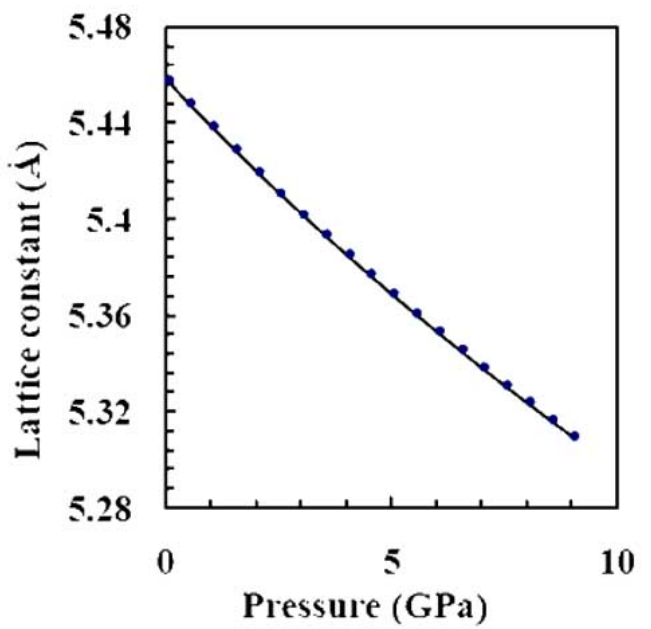

Fig. (3). The effect of pressure on the lattice constant of AlP.

The pressure dependence of the bulk modulus and the cohesive energy is illustrated in Figs. (4 and 5), respectively. It is shown that the bulk modulus increases linearly with the pressure. On the other hand, the absolute value of the cohesive energy decreases as the pressure increases.

The effect of pressure on the high symmetry points $\left(\Gamma_{1 \mathrm{v}}\right.$, $\Gamma_{15 \mathrm{v}}, \mathrm{X}_{1 \mathrm{v}}, \mathrm{X}_{5 \mathrm{v}}, \mathrm{X}_{1 \mathrm{c}}, \mathrm{X}_{5 \mathrm{c}}, \Gamma_{15 \mathrm{c}}$, and $\Gamma_{1 \mathrm{c}}$ ) is shown in Fig. (6). From this figure one can notice that the eigenvalues at conduction band $\left(\mathrm{X}_{5 \mathrm{c}}, \Gamma 1_{5 \mathrm{c}}, \Gamma_{1 \mathrm{c}}, \mathrm{X}_{1 \mathrm{c}}\right)$ increase with pressure, whereas eigenvalues at valence band $\left(X_{5 v}, X_{1 v}, \Gamma_{1 v}\right)$ decrease with pressure, However, the decrease of $X_{5 \mathrm{v}}, X_{1 \mathrm{v}}$, and $\Gamma_{1 \mathrm{v}}$ with pressure is small.

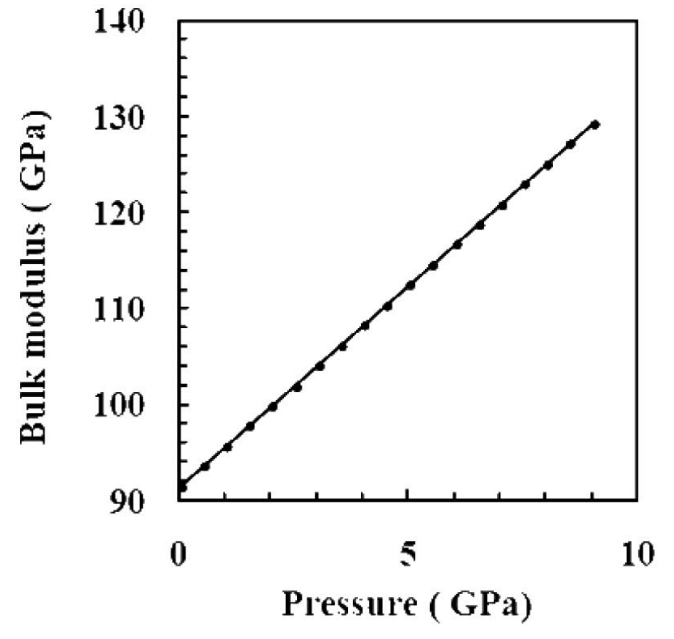

Fig. (4). The bulk modulus as a function of pressure for AlP.

Fig. (7) shows the pressure dependence of the indirect band gap of the $\mathrm{zb}$ phase of AlP from the present energy band structure calculations. The indirect bandgap increases with the increase of pressure; because the minimum conduction energy level rises and the top valence energy level lowers with the increase of pressure. However, in most cases the first pressure-induced phase transition corresponds to the closing of the bandgap and metallization of the sample. In the present work, the pressure derivative of the indirect bandgap is computed to be $\sim 4.2 \mathrm{meV} / \mathrm{GPa}$.

The predicted effect of pressure on the conduction bandwidth and valence bandwidth is illustrated in Fig. (8). The 
conduction bandwidth decreases with the increase of pressure, while the valence bandwidth increases with the increase of pressure. Our calculations give a pressure derivative of $\sim$ $1.9 \mathrm{meV} / \mathrm{GPa}$ for the conduction bandwidth, and 22 $\mathrm{meV} / \mathrm{GPa}$ for the valence bandwidth.

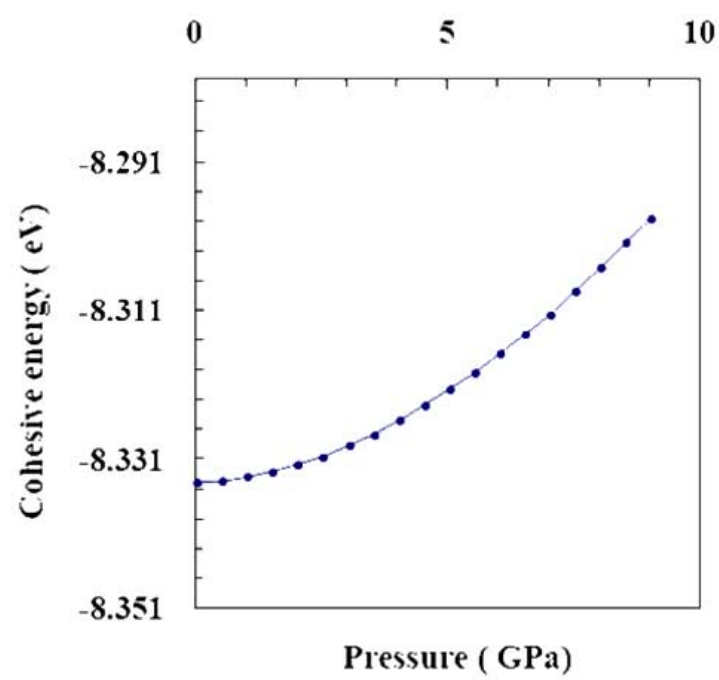

Fig. (5). The effect of pressure on the cohesive energy of AlP.

\section{CONCLUSIONS}

In this paper, a study of some properties of AlP is presented. The cohesive energy, lattice constant, bulk modulus, and its pressure derivative have been calculated by (LUCCNDO) method. The calculated results indicate that this model gives results in good agreement with the corresponding experimental results, and this shows the possibility of using this model in qualitative study of some materials. The LUC-CNDO method is shown to be time efficient and retains many of the essential features of ab initio HartreeFock theory. A reasonable agreement of the valence band- width is shown in comparison with the available theoretical result. However, there is a large difference between the calculated indirect band gap and the corresponding experimental value. The effect of pressure on these properties is investigated. It is found that the conduction bandwidth decreases with increasing the pressure, whereas the indirect bandgap, valence bandwidth, and cohesive energy increase with the increase of pressure. The maximum value of pressure is taken to be $9 \mathrm{GPa}$, because beyond this value of pressure, the phase of AlP transforms from zb to rock salt phase. Relativistic effect is added to the calculation of the band gap, also zero point energy is added to the calculation of the cohesive energy. Finally, this model is shown to give a good description to the charge density of AlP and it is expected that this method could give reliable description for other materials that have zinc blende and cubic structures.

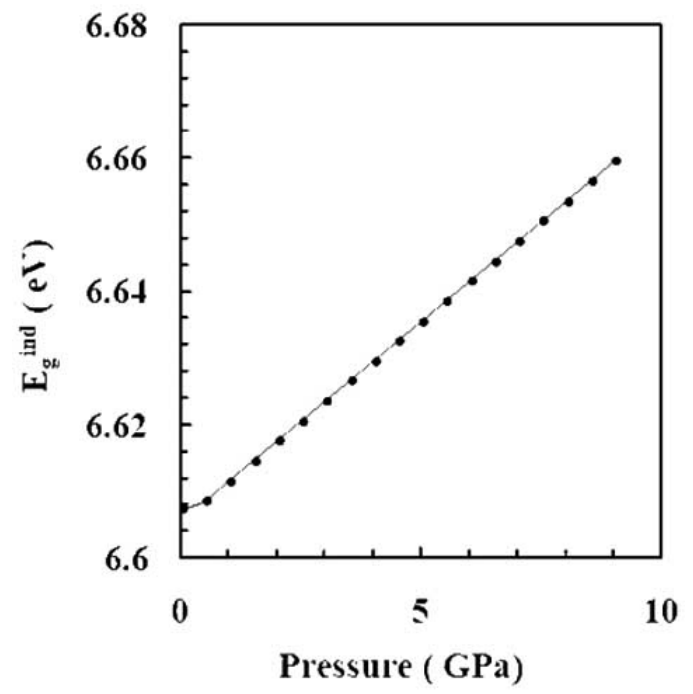

Fig. (7). Variation of the indirect band gap versus pressure for AlP.

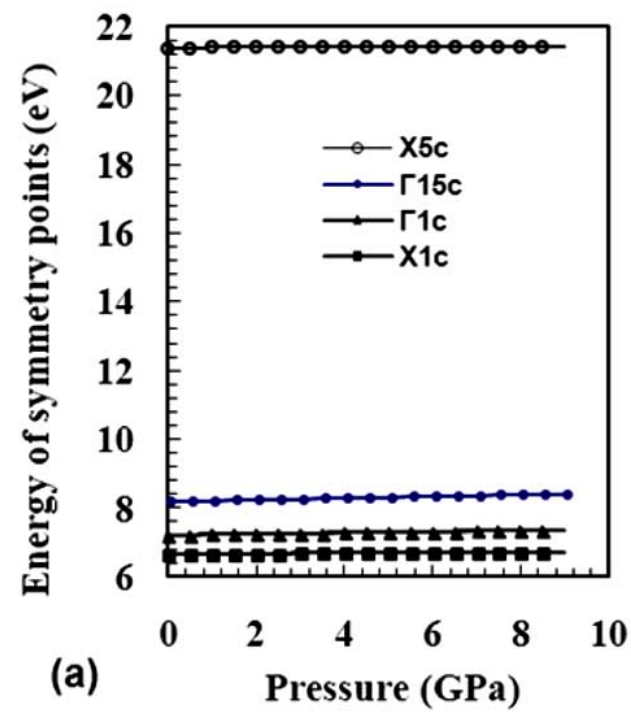

Fig. (6). The effect of pressure on the high symmetry points in the (a) conduction band $\left(X_{5 c}, \Gamma_{15 c}, \Gamma_{1 c}, X_{1 c}\right)$, and (b) valence band $\left(\mathrm{X}_{5 \mathrm{v}}, \mathrm{X}_{1 \mathrm{v}}, \Gamma_{1 \mathrm{v}}\right)$. 

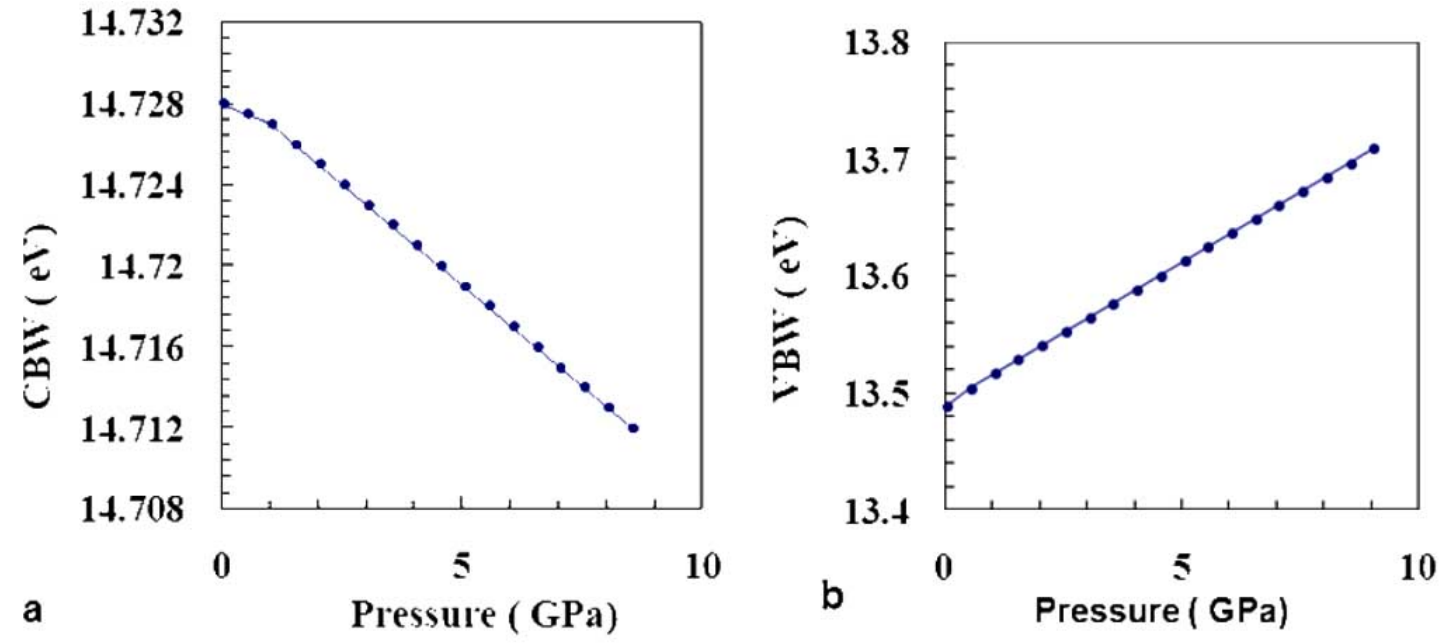

Fig. (8). Effect of pressure on the (a) conduction bandwidth, and (b) valence bandwidth.

\section{REFERENCES}

[1] Greene RG, Luo H, Ruoff AL. High pressure study of AlP: Transformation to a metallic NiAs phase. J Appl Phys 1994; 76: 7296305.

[2] Mujica A, Rodríguez-Hernández P, Radescu S, Needs RJ, Muñoz A. AlX $(\mathrm{X}=\mathrm{As}, \mathrm{P}, \mathrm{Sb})$ Compounds under Pressure. Phys Stat Solidi (b) 1999; 211: 39-43.

[3] Baublitz M, Ruoff AL. Diffraction studies of the high pressure phases of GaAs and GaP. J Appl Phys 1982; 53: 6179- 86.

[4] Zhang SB, Cohen NL. High-pressure phases of III-V zinc-blende semiconductors. Phys Rev B 1987; 35: 7604-10.

[5] Mujica A, Rubio A, Munoz A, Needs RJ. High-pressure phases of group-IV, III-V, and II-VI compounds. Rev Mod Phys 2003; 75: 863-912.

[6] Froyen S, Cohen ML. Structural properties of III-V zinc-blende semiconductors under pressure. Phys Rev B 1983; 28: 3258-65.

[7] Jivani AR, Trivedi HJ, Gajjar PN, Jani AR. Total energy, equation of state and bulk modulus of AlP, AlAs and AlSb semiconductors. Parmana-J Phys 2005; 64: 153-8.

[8] Ackland GJ. High-pressure phases of group IV and III-V semiconductors. Rep Prog Phys 2001; 64: 483-516.

[9] Andriyevsky B, Ciepluch-Trojanek W, Patryn A. Effect of hydrostatic pressure on structural and electronic properties of TGS crystals (first-principle calculations). Condens Matter Phys 2007; 10:33-8.

[10] Harker AH, Larkins FP. A large unit cell semiempirical molecular orbital approach to the properties of solids. I. General theory. J Phys C : Solid State Phys 1979; 12: 2487-95.

[11] Abdulsattar MA, Al-Bayati KH. Corrections and parametrization of semiempirical large unit cell method for covalent semiconductors. Phys Rev B 2007; 75: 245201-9.

[12] Radi IO, Abdulsattar MA, Abdul-Lettif AM. Semiempirical LUCINDO calculations on the effect of pressure on the electronic structure of diamond. Phys Stat Solidi (b) 2007; 244 (4): 1304-17.

[13] Pople J, Beveridge D. Approximate MO Theories. New York; McGraw-Hill: 1970.
[14] Evarestov R, Lovchikov V. Large unit cell calculations of solids in the CNDO approximation. Phys Stat Solidi (b) 1997; 79: 743-51.

[15] Szymanski JE. Semi-empirical methods of total energy calculations. Ph. D. thesis, Canada: University of York 1984.

[16] Lee S, Kang J, Kang M. Structural properties of semiconductors in the generalized gradient approximation. J Korean Phys Soc 1997; 31: 811-9.

[17] Pässler R. Parameter sets due to fittings of the temperature dependencies of fundamental bandgaps in semiconductors. Phys Stat Solidi (b) 1999; 216: 975-1007.

[18] Causa M, Zupan A. Density functional LCAO calculation of periodic systems. A posteriori correction of the Hartree-Fock energy of covalent and ionic crystals. Chem Phys Lett 1994; 220: 145-52.

[19] Ahmed R, Aleem F, Hashemifar SJ, Akbarzadeh H. First-principles study of the structural and electronic properties of III-phosphides. Phys B: Condens Matter 2008; 403: 1876-81.

[20] Murnghan FD. The compressibility of media under extreme pressures. Proc Natl Acad Sci USA 1944; 3: 244-7.

[21] Vurgaftman I, Meyer JR, Ram-Mohan LR. Band parameters for III-V compound semiconductors and their alloys. J Appl Phys 2001; 89: 5815-22.

[22] Shishkin M, Kresse G. Self-consistent GW calculations for semiconductors and insulators. Phys Rev B 2007; 75: 235102-10.

[23] Chow PC, Liu L. Relativistic effects on the electronic band structure of compound semiconductors. Phys Rev 1965; 140: 1817 26.

[24] Lawaetz P. Valence-band parameters in cubic semiconductors. Phys Rev B 1971; 4: 3460-7.

[25] Fuchs F, Furthmüller J, Bechstedt F. Quasiparticle band structure based on a generalized Kohn-Sham scheme. Phys Rev B 2007; 76: 115109-16.

[26] Wang SQ, Ye HQ. Ab initio investigation of the pressure dependences of phonon and dielectric properties for IIIV semiconductors. J Phys: Condens Matter 2005; 17: 4475-88.

[27] Baublitz M, Ruoff AL. Diffraction studies of the high pressure phases of GaAs and GaP. J Appl Phys 1982; 53: 6179- 86. 